\title{
Minimal time control of fed-batch bioreactor with product inhibition
}

\author{
Térence Bayen • Francis Mairet
}

September 21, 2012

\begin{abstract}
This paper is devoted to the minimal time control problem for fed-batch bioreactors, in presence of an inhibitory product, which is released by the biomass proportionally to its growth. We first consider a growth rate with substrate saturation and product inhibition, and we prove that the optimal strategy is fill and wait (bang-bang). We then investigate the case of the Jin growth rate which takes into account substrate and product inhibition. For this type of growth function, we can prove the existence of singular arc paths defining singular strategies. Several configurations are addressed depending on the parameter set. For each case, we provide an optimal feedback control of the problem (of type bang-bang or bang-singular-bang). These results are obtained gathering the initial system into a planar one by using conservation laws. Thanks to Pontryagin maximum principle, Green's theorem, and properties of the switching function, we obtain the optimal synthesis. A methodology is also proposed in order to implement the optimal feeding strategies.
\end{abstract}

Keywords Optimal control · Fedbatch bioprocess · Jin growth rate

\section{T. Bayen}

Université Montpellier 2, Case courrier 051, 34095 Montpellier cedex 5, France.

INRA-INRIA 'MODEMIC' team, INRIA Sophia-Antipolis Méditerranée, UMR INRA-SupAgro 729 'MISTEA' 2 place Viala 34060 Montpellier.

E-mail: tbayen@math.univ-montp2.fr

F. Mairet

Departamento de Matemática, Universidad Técnica Federico Santa María, Avda España 1680, Valparaíso, Chile.

Tel.: +56322654785

Fax: +56 322654563

E-mail: francis.mairet@usm.cl

\section{Introduction}

Fed-batch operation of bioreactor is a popular operating mode used in industry as the limiting substrate concentration can be easily controlled, see e.g. [1]. Moreover, it allows to reach a high concentration of cells or products, or a low concentration of substrate (for depollution). Defining an optimized feeding strategy is a real challenge which can be tackled using optimal control theory (see e.g. [2]). For the minimal time problem (i.e. given initial conditions, the goal is to define a feeding policy in order to reach a given substrate concentration with a completely full reactor in a minimal amount of time), the optimal synthesis (that is the description of an optimal feedback control for any initial condition) has been proposed by [3] for increasing growth functions (e.g., the Monod kinetic, see [4,5]) and nonmonotonic growth functions with one maximum point (e.g., Haldane kinetic, see $[6,5]$ ) using Green's theorem, via the technique introduced in [7]. More recently, the problem for growth functions with two local maxima has been tackled numerically [8] and analytically [9] allowing impulsive controls (corresponding to instantaneous dilutions, see [10]).

In this paper, we consider the minimal time control problem for fed-batch bioreactors in presence of an inhibitory product. Optimal control problems with product inhibition have been tackled by [11] using Kelley's transformation [12] for specific rate of product formation which are not correlated to the specific growth rate. As an example, the optimal feeding strategy to maximize the amount of ethanol produced by Saccharomyces cerevisiae is provided. Contrary to [11], we consider in this work that the product is released by the biomass proportionally to its growth [13]. The growth rate function associated to this model is a smooth func- 
tion $\mu(s, p)$ depending both on the substrate and product concentrations.

The paper is organized as follows. In Section 2, the problem is stated, and we derive several general properties about extremal trajectories via the Pontryagin maximum principle. In Section 3, we give the optimal strategy for the case of an inhibition by product only and Section 4 is devoted to the case of an inhibition by product and substrate. As an example, we provide the optimal synthesis for the Jin growth rate [14]. In Section 5, we propose a method in order to implement the optimal strategies. Finally, in Section 6, we discuss a controllability assumption which was previously used.

\section{Statement of the problem and general results}

\subsection{Formulation of the problem}

A perfectly mixed bioreactor with product inhibition operated in fed-batch can be described by the following system (after a scaling):

$$
\left\{\begin{array}{l}
\dot{x}=\left(\mu(s, p)-\frac{u}{v}\right) x, \\
\dot{s}=-\mu(s, p) x+\frac{u}{v}\left(s_{i n}-s\right), \\
\dot{p}=\mu(s, p) x-\frac{u}{v} p, \\
\dot{v}=u,
\end{array}\right.
$$

where $x, s$, and $p$ are respectively the concentrations of biomass, substrate, and product, and $v$ is the volume of the tank. Here $u$ is the input flow (which is a measurable control function taking values in $[0,1])$ and $s_{i n}$ is the concentration of substrate in the input flow. If $(x, s, p, v)$ is a solution of (1), one can see that the functions $M:=v\left(x+s-s_{i n}\right)$ and $N:=v\left(p+s-s_{i n}\right)$ are constant. Therefore, we have $x=\frac{M}{v}-s+s_{i n}$ and $p=\frac{N}{v}-s+s_{i n}$, and the system can be gathered into a planar system with a drift and a single input $u$ :

$$
\left\{\begin{array}{l}
\dot{s}=-h(s, v)\left(\frac{M}{v}-s+s_{i n}\right)+\frac{u}{v}\left(s_{i n}-s\right), \\
\dot{v}=u
\end{array}\right.
$$

where $h(s, v):=\mu\left(s, \frac{N}{v}-s+s_{i n}\right)$. Note that for $N=$ 0 , the system can be written with a growth function $h_{0}(s):=\mu\left(s,-s+s_{i n}\right)$ depending only on the substrate concentration.

The optimal control problem can be stated as follows. We aim at finding a feeding strategy (that is a control $u(\cdot))$ steering System (2) in a minimal amount of time $t_{f}(u)$ to a given target $\mathcal{T}$ :

$\inf _{u \in \mathcal{U}} t_{f}(u)$, s.t. $\xi\left(t_{f}(u)\right) \in \mathcal{T}$, where $\xi(\cdot):=(s(\cdot), v(\cdot))$, and $\mathcal{U}$ is the set of admissible controls $u$. In the present work, $\mathcal{T}$ is given by:

$\mathcal{T}=\left\{\xi \in \mathbb{R}_{+}^{2} \mid\right.$ s.t. $\left.s\left(t_{f}\right) \leq s_{\text {ref }}, v\left(t_{f}\right)=v_{m}\right\}$,

where $s_{r e f}$ is a given substrate concentration, and $v_{m}$ is the volume of the tank. This set is of particular interest for wastewater treatment.

Given the domain $\mathcal{D}=\left[0, s_{i n}\right) \times\left(0, v_{m}\right]$, one can prove that the target can be reached from any initial condition $\left(s_{0}, v_{0}\right) \in \mathcal{D}$ by taking $u=1$ until $v=v_{m}$ and then applying $u=0$ until $s \leq s_{\text {ref }}$ if necessary. The existence of an optimal control is standard by applying Fillipov's Theorem, see [15]. In the following, we call $\mathcal{P}$ the optimal control problem (2)-(3), with initial condition $\left(s_{0}, v_{0}\right) \in \mathcal{D}$, and we apply Pontryagin maximum principle (PMP) on $\mathcal{P}$.

\subsection{Pontryagin maximum principle}

Let $H=H\left(s, v, \lambda_{s}, \lambda_{v}, \lambda_{0}, r, u\right)$ the Hamiltonian of the system:

$$
\begin{aligned}
H=-\lambda_{s} h(s, v) & {\left[\frac{M}{v}-\left(s-s_{i n}\right)\right] } \\
& +u\left[\frac{\lambda_{s}\left(s_{i n}-s\right)}{v}+\lambda_{v}\right]+\lambda_{0}
\end{aligned}
$$

If $u$ denotes an optimal control and $(s, v)$ the corresponding solution of (2), there exists $t_{f}>0, \lambda_{0} \leq$ 0 , and an absolutely continuous map $\lambda=\left(\lambda_{s}, \lambda_{v}\right)$ : $\left[0, t_{f}\right] \rightarrow \mathbb{R}^{2}$ such that $\left(\lambda_{0}, \lambda\right) \neq 0, \dot{\lambda}_{s}=-\frac{\partial H}{\partial s}, \dot{\lambda}_{v}=$ $-\frac{\partial H}{\partial v}$, that is:

$\left\{\begin{array}{l}\dot{\lambda}_{s}=\lambda_{s}\left(\frac{\partial h(s, v)}{\partial s} x-h(s, v)+\frac{u}{v}\right), \\ \dot{\lambda}_{v}=\lambda_{s}\left(\frac{\partial h(s, v)}{\partial v} x+\frac{-h(s, v) M+u\left(s_{i n}-s\right)}{v^{2}}\right),\end{array}\right.$

and we have the maximality condition:

$$
u(t) \in \operatorname{argmax}_{\omega \in[0,1]} H\left(s(t), v(t), \lambda_{s}(t), \lambda_{v}(t), \lambda_{0}, \omega\right),
$$

for almost every $t \in\left[0, t_{f}\right]$. We call extremal trajectory a sextuplet $\left(s(\cdot), v(\cdot), \lambda_{0}, \lambda_{s}(\cdot), \lambda_{v}(\cdot), u(\cdot)\right)$ satisfying (2)(6)-(7), and extremal control the control $u$ associated to this extremal trajectory. As $t_{f}$ is free, the Hamiltonian is zero along an extremal trajectory. Notice from (5)-(6) that $\lambda_{s}$ is always non-zero (it is therefore of constant sign, see also Lemma 1).

Next, let us define the switching function $\phi$ associated to the control $u$ by:

$\phi:=\lambda_{s} \frac{s_{i n}-s}{v}+\lambda_{v}$. 
We obtain from (7) that any extremal control satisfies the following control law: for a.e. $t \in\left[0, t_{f}\right]$, we have

$$
\left\{\begin{array}{lll}
\phi(t)<0 & \Longrightarrow u(t)=0 & \\
\phi(t)>0 & \Longrightarrow u(t)=1 \\
\phi(t)=0 \Longrightarrow u(t) \in(0,1] & & \text { (No feeding) }
\end{array}\right.
$$

If $\phi$ vanishes in an isolated point $t_{0}$, then $u$ is bangbang around $t_{0}$ (that is $u$ switches from 0 or 1 to another extremal value 0 or 1 at time $\left.t_{0}\right)$. Whenever $\phi$ is zero on some time interval $I \subset\left[0, t_{f}\right](\operatorname{such}$ that meas $(I)>0)$, we say that $u$ is a singular control, and the trajectory contains a singular arc (see e.g. [17]). A computation shows that we have

$\dot{\phi}=\lambda_{s} x \psi(s, v)$

with

$\psi(s, v)=\frac{s_{i n}-s}{v} \frac{\partial h}{\partial s}(s, v)+\frac{\partial h}{\partial v}(s, v)$.

It follows that if $I$ is a singular arc, we have $\dot{\phi}=0$, on $I$, that is:

$\psi \equiv 0$,

as $\lambda_{s}$ and $x$ are non-zero.

The sign of $\lambda_{s}$ is of particular interest in order to study the switching function. Following [8], let us consider the curve $v \longmapsto \gamma(v)$ which is the unique solution of (2) with constant control $u=1$ and that passes through $\left(s_{r e f}, v_{m}\right)$. Moreover consider:

$\mathcal{D}^{\prime}=\{(s, v) \in \mathcal{D} \quad \mid s \geq \gamma(v)\}$

Lemma 1 If $\left(s_{0}, v_{0}\right)$ is in $\mathcal{D}^{\prime}$, then any optimal trajectory satisfies $\lambda_{s}<0$.

Proof From System (6), we have that if $\lambda_{s}(0)=0$, then $\lambda_{s}(t)$ is always zero, and if $\lambda_{s}(0) \neq 0$, then $\lambda_{s}(t)$ is always non-zero and of constant sign. An optimal trajectory is a concatenation of arcs where $u=0$ (no feeding), $u=1$ (maximal feeding), or singular arcs satisfying $\phi=0$. If the initial condition is in $\mathcal{D}^{\prime}$, any optimal trajectory contains at least an arc $u=0$ or a singular arc (otherwise, the trajectory would not reach $\mathcal{T}$ ). Consequently, there exists an interval $\left[t_{1}, t_{2}\right]$ such that on this interval, one has:

$$
H=-\lambda_{s} h(s, v)\left[\frac{M}{v}-\left(s-s_{i n}\right)\right]+\lambda_{0}=0
$$

Now, if at some point $t$, we have $\lambda_{s}(t)>0$, we get a contradiction as $\lambda_{0} \leq 0$ and $x=\frac{M}{v}-\left(s-s_{i n}\right)>0$. Hence, we have $\lambda_{s}<0$.
By a similar argument as in the proof of the previous lemma, one can prove immediately that $\lambda_{0} \neq 0$. By homogeneity, we take $\lambda_{0}=-1$ in the following.

The next proposition allows to compare the cost of two trajectories and is based on Green's Theorem and a clock form argument, see $[7,3]$.

Proposition 1 Consider two points $A$ and $B$ in $\mathcal{D}$, and two different trajectories $T_{a}$ and $T_{b}$ joining $A$ to $B$, such that the trajectory $T_{b}$ from $A$ to $B$ followed by the trajectory $T_{a}$ from $B$ to $A$ is a positively oriented curve $\Gamma$. Let $\mathcal{A}$ be the region enclosed by $\Gamma$. If $\psi(s, v) \geq$ 0 (resp. $\leq 0)$ for all $(s, v) \in \mathcal{A}$, then the cost $J_{a}$ of trajectory $T_{a}$ is bigger (resp. lower) than the cost $J_{b}$ of trajectory $T_{b}$.

Proof Using Green's Theorem, we obtain:

$J_{b}-J_{a}=\oint_{\Gamma} d t=\iint_{\mathcal{A}}-\frac{\psi(s, v)}{\mu(s, p)^{2} x} d s d v$.

If $\psi \geq 0$ (resp. $\psi \leq 0$ ), it follows that $J_{b}-J_{a} \leq 0$ (resp. $\left.J_{b}-J_{a} \geq 0\right)$ from the integral above, which proves the result (see [7] and [3] for more details).

The expression of $\psi(s, v)$ will be important in the following in order to apply this proposition. We first express $\psi$ in term of the growth function $\mu$. In the following expression, we have written $p$ in instead of $p(v)=$ $\frac{N}{v}-s+s_{i n}$. By derivating, we get

$$
\frac{\partial h}{\partial s}(s, v)=\frac{\partial \mu}{\partial s}(s, p)+\frac{\partial p}{\partial s} \frac{\partial \mu}{\partial p}(s, p)=\left[\frac{\partial \mu}{\partial s}-\frac{\partial \mu}{\partial p}\right](s, p)
$$

and:

$$
\frac{\partial h}{\partial v}(s, v)=\frac{\partial p}{\partial v} \frac{\partial \mu}{\partial p}(s, p)=-\frac{N}{v^{2}} \frac{\partial \mu}{\partial p}(s, p) .
$$

Thus, $\psi$ can be written:

$\psi(s, v)=\frac{s_{i n}-s}{v} \frac{\partial \mu}{\partial s}(s, p)-\frac{N+v\left(s_{i n}-s\right)}{v^{2}} \frac{\partial \mu}{\partial p}(s, p)$.

The previous expression will be used in order to compute singular arcs in Section 4.

\subsection{Computation of singular arcs}

In this part, we provide an expression of singular controls in the general case of System (2) and we discuss the admissibility of singular arcs. The computation of singular controls is based on the second derivative of $\phi$ 
which can be obtained by using Lie brackets, see e.g. [16]. A direct computation shows that:

$$
\begin{aligned}
\ddot{\phi} & =\lambda_{s} x\left\{u \left[\frac{\partial^{2} h}{\partial v^{2}}+2 \frac{\left(s_{i n}-s\right)}{v}\left(\frac{\partial^{2} h}{\partial s \partial v}-\frac{1}{v} \frac{\partial h}{\partial s}\right)\right.\right. \\
& \left.\left.+\frac{\left(s_{i n}-s\right)^{2}}{v^{2}} \frac{\partial^{2} h}{\partial s^{2}}\right]-h x \frac{\partial^{2} h}{\partial s \partial v}-\frac{1}{v} \frac{\partial h}{\partial s}+\frac{s_{i n}-s}{v} \frac{\partial^{2} h}{\partial s^{2}}\right\} .
\end{aligned}
$$

To address the optimality of a singular arc, we use Legendre-Clebsch necessary condition, see e.g. [17]. If $u$ is a singular optimal control, we must have:

$\frac{\partial}{\partial u} \frac{d^{2}}{d t^{2}} H_{u} \geq 0$,

along the singular arc, where $H_{u}=\phi$. In this framework, Legendre-Clebsch condition writes:

$\frac{\partial^{2} h}{\partial v^{2}}+2 \frac{\left(s_{i n}-s\right)}{v}\left(\frac{\partial^{2} h}{\partial s \partial v}-\frac{1}{v} \frac{\partial h}{\partial s}\right)+\frac{\left(s_{i n}-s\right)^{2}}{v^{2}} \frac{\partial^{2} h}{\partial s^{2}} \geq 0$.

Moreover, if $\frac{\partial}{\partial u} \frac{d^{2}}{d t^{2}} H_{u}>0$ along the singular arc, the singular control is given by:

$\tilde{u}(s, v)=h x \frac{\frac{\partial^{2} h}{\partial s \partial v}-\frac{1}{v} \frac{\partial h}{\partial s}+\frac{s_{i n}-s}{v} \frac{\partial^{2} h}{\partial s^{2}}}{\frac{\partial^{2} h}{\partial v^{2}}+2 \frac{\left(s_{i n}-s\right)}{v}\left[\frac{\partial^{2} h}{\partial s \partial v}-\frac{1}{v} \frac{\partial h}{\partial s}\right]+\frac{\left(s_{i n}-s\right)^{2}}{v^{2}} \frac{\partial^{2} h}{\partial s^{2}}}$.

Now, we address the question of admissibility of a singular arc. First, notice that if for all $\left(s_{0}, v_{0}\right) \in \mathcal{D}^{\prime}$, we have

$$
\frac{\partial \psi}{\partial v}\left(s_{0}, v_{0}\right) \neq 0,
$$

then by the implicit function Theorem, there exists a unique function $s \longmapsto \tilde{v}(s)$, defined in some neighborhood $\mathcal{W}$ of $s_{0}$ and such that $\psi(s, \tilde{v}(s))=0$ for all $s \in \mathcal{W}$.

Therefore, if we consider a singular arc defined on some time interval $\left[t_{1}, t_{2}\right]$, we have that it is admissible if and only if

$$
\left\{\begin{array}{l}
\tilde{v}(s(t))>0, \forall t \in\left[t_{1}, t_{2}\right], \\
\tilde{u}(s(t), \tilde{v}(s(t))) \in[0,1], \forall t \in\left[t_{1}, t_{2}\right] .
\end{array}\right.
$$

In Section 4, the first condition of (18) is used in order to determine the structure of an optimal control. Moreover, we assume in section 4 that the singular arc is controllable, that is the singular control $\tilde{u}$ takes values in $[0,1]$. In Section 6 , we will discuss the validity of this assumption.

In the case where $h(s, v)=h_{0}(s)$, condition (11) implies that

$h_{0}^{\prime}(s)=0$, hence the concentration of substrate $s(t)$ is constant and is equal to a critical point $\tilde{s}$ of $h_{0}$ (if it exists). Moreover, we have in in this case

$$
\frac{\partial}{\partial u} \frac{d^{2}}{d t^{2}} H_{u}=\lambda_{s} x \frac{s_{i n}-s}{v^{2}} h^{\prime \prime}(s),
$$

hence, (15) implies that only local maxima of $h_{0}$ are candidates for optimality (see $[9],[8]$ ).

\section{Inhibition by product only}

In this section, we study problem $\mathcal{P}$ in the case of inhibition by the product which means that the mapping $s \longmapsto \mu(s, p)$ is increasing with respect to $s$ for all $p$, and that the mapping $p \longmapsto \mu(s, p)$ is decreasing with respect to $p$ for all $s>0$.

Property 1 In the case of inhibition by product only, the optimal strategy is fill and wait.

Proof Since $N+v\left(s_{i n}-s\right)=v p \geq 0$, we get from (14) that $\psi(s, v)>0$ for all $(s, v) \in \mathcal{D}$. Therefore, $\dot{\phi}(t) \neq 0$, so the optimal strategy does not contain a singular arc. Using Proposition 1, we can conclude that the optimal strategy is $u=1$ until $v_{m}$, and then $u=0$ (strategy fill and wait).

Remark 1 Using the same approach, we can show that this strategy is also optimal for bioprocesses in which microbial growth is represented by the Contois model $\mu(s, x)$, see [18]:

$$
\mu(s, x)=\mu_{m} \frac{s}{k x+s} .
$$

In particular $\mu$ is increasing with respect to $s$ and decreasing with respect to $x$. This growth rate is widely used in wastewater treatment as it is suitable to represent hydrolysis, which is generally the limiting step for particulate waste treatment.

\section{Inhibition by product and substrate}

In this section, we consider inhibition by product and substrate, using as an example the growth rate proposed by Jin et al. (see [14]):

$\mu(s, p)=\mu_{m} \frac{s}{k_{1}+s} e^{-k_{2} p-k_{3} s}$.

Notice that $s \longmapsto \mu(s, p)$ is non-monotonic. In the case where $\mu$ is given by (20), we obtain by (14):

$\psi(s, v)=\mu_{m} \frac{e^{-k_{2} p-k_{3} s}}{v\left(k_{1}+s\right)} \Psi(s, v)$, 
with:

$$
\Psi(s, v)=\left(s_{\text {in }}-s\right)\left[\frac{k_{1}}{k_{1}+s}+s\left(k_{2}-k_{3}\right)\right]+\frac{k_{2} N s}{v} .
$$

This expression will allow to characterize singular arcs in the next section.

\subsection{Case $N=0$}

For future reference, let us define a polynomial $\rho$ by

$$
\rho(s)=s^{2}+k_{1} s+\frac{k_{1}}{k_{2}-k_{3}} .
$$

The discriminant of $\rho$ reads: $\Delta=k_{1}\left(k_{1}-\frac{4}{k_{2}-k_{3}}\right)$, and whenever $\Delta \geq 0$, let $\bar{s}=\frac{-k_{1}+\sqrt{\Delta}}{2}$ the positive root of $\rho$. Notice that we always have $\rho^{\prime} \geq 0$.

From (19), $s=\bar{s}$ is a singular arc provided that $\Delta \geq 0$ (see also [9],[8],[3]), and we can define a singular control $\bar{u}$ depending only on $v$ and which is obtained solving (2) when the substrate concentration is constant equal to $\bar{s}$ :

$\bar{u}(v)=h_{0}(\bar{s})\left(v+\frac{M}{s_{i n}-\bar{s}}\right)$.

The singular arc is therefore admissible provided that $\bar{u}(v) \in[0,1]$ for all $v \in\left(0, v_{m}\right]$. First, one has $x=$ $\frac{M}{v}+s_{i n}-s>0$ so that $\bar{u}(v)>0$. Thus, if:

$h_{0}(\bar{s})\left(v_{m}+\frac{M}{s_{i n}-\bar{s}}\right)<1$,

then the singular arc is admissible. This assumption is generally used for minimal time control of fed-batch reactor with nonmonotonic growth rate (see e.g. [10]). The next proposition gives an optimal synthesis of the problem for $N=0$ which is closely related to the one obtained in [3].

Proposition 2 Assume that (23) is satisfied.

(i). If $\Delta \leq 0$ or $\bar{s} \geq s_{i n}$, the optimal strategy is fill and wait (bang-bang).

(ii). If $\Delta>0$ and $\bar{s}<s_{\text {in }}$, the optimal strategy is the singular arc strategy $\bar{s}$, defined as follows (see Fig. 1):

$$
u\left(s_{0}, v_{0}\right)=\left\{\begin{array}{l}
\bar{u} \text { if } s_{0}=\bar{s} \text { and } v_{0}<v_{m}, \\
0 \text { if } s>\bar{s} \text { or } v_{0}=v_{m} \\
1 \text { if } s<\bar{s} \text { and } v_{0}<v_{m}
\end{array}\right.
$$

where control $\bar{u}$ is given by (22) and is such that $s(t)=\bar{s}$ until the volume reaches $v_{m}$.
Proof If $N=0$, it follows from (21) that a singular arc is possible if $\rho(s)=0$. If $\Delta \leq 0$, the equation $\rho=0$ does not have any positive root, so a singular arc is not possible, and $\psi(s, v)>0$ for all $(s, v) \in \mathcal{D}$. If $\Delta>0$, there exists a positive root $\bar{s}$ of $\rho=0$ which defines a singular arc, and $\psi(s, v)>0$ (resp. $<0$ ) if $s<\bar{s}$ (resp. $>\bar{s})$. Using Proposition 1, we can conclude that the optimal strategy is:

- fill and wait if $\Delta \leq 0$ or $\bar{s} \geq s_{\text {in }}$,

- the singular arc strategy $\bar{s}$ if $\Delta>0$ and $\bar{s}<s_{\text {in }}$.

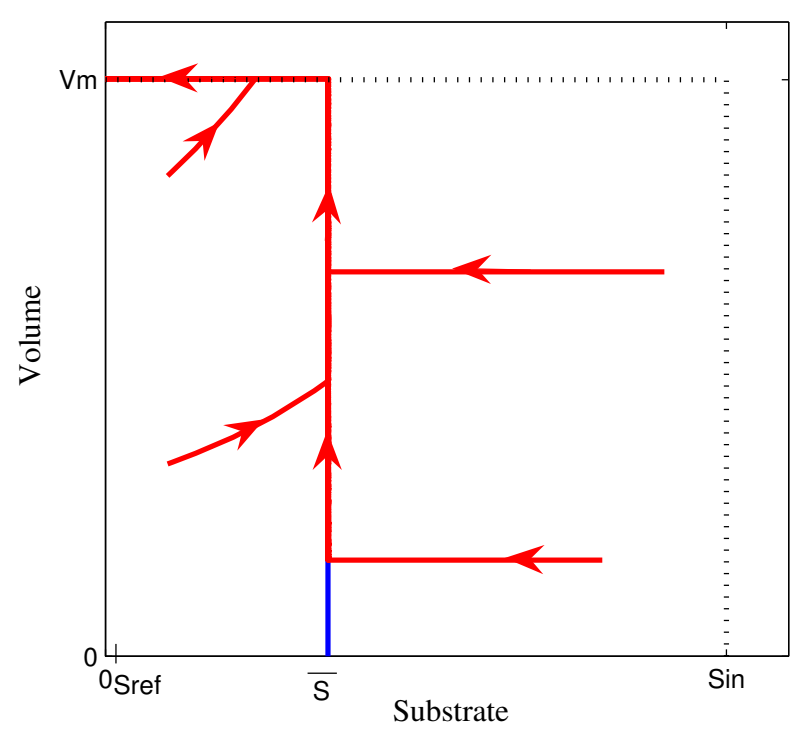

Fig. 1 Optimal trajectories (in red) for various initial conditions for the Jin growth rate with $N=0$ (see Proposition 2). In blue, the line $s=\bar{s}$. Parameter values used for simulation are given in Table 1.

4.2 Computation of singular arcs in the case $N \neq 0$

We now investigate the case where the parameter $N$ is non-zero. First, let us characterize the singular arc in this case. From (21), we obtain that along a singular arc, the volume $\tilde{v}$ depends on the concentration $s$ by:

$\tilde{v}(s)=-N \frac{s\left(k_{1}+s\right) k_{2}}{\left(s_{\text {in }}-s\right)\left[k_{1}+s\left(k_{1}+s\right)\left(k_{2}-k_{3}\right)\right]}$.

Remark 2 One has $\Psi(s, v)=\frac{k_{2} N s}{v}\left(1-\frac{v}{\tilde{v}(s)}\right)$ for all $(s, v) \in \mathcal{D}^{\prime}$. 
The derivative of $\tilde{v}(s)$ writes:

$\frac{\partial \tilde{v}}{\partial s}=\frac{\tilde{v}(s)}{s_{i n}-s}-\frac{N k_{1} k_{2}\left(k_{1}+2 s\right)}{\left(s_{i n}-s\right)\left[k_{1}+s\left(k_{2}-k_{3}\right)\left(k_{1}+s\right)\right]^{2}}$.

By combining the previous equality and (2), we get that the singular control only depends on the substrate concentration and is given by:

$\tilde{u}(s)=\frac{x(s) \tilde{v}(s) h(s, \tilde{v}(s))}{s_{i n}-s}\left(1-\tilde{v}(s) \frac{\left(k_{2}-k_{3}\right)^{2}}{N k_{1} k_{2}} \frac{\rho^{2}(s)}{\rho^{\prime}(s)}\right)$

where $x(s)=\frac{M}{\tilde{v}(s)}+\left(s_{i n}-s\right)$.

The next lemma is concerned with the orientation of the singular arc.

Lemma 2 If $N<0$ (resp. $N>0$ ), then $\dot{s}>0$ (resp. $\dot{s}<0$ ) along the singular arc, and the singular arc is oriented clockwise (resp. counterclockwise).

Proof Replacing the expression of the singular control $\tilde{u}(s)$ into $(2)$ yields to:

$$
\dot{s}=-\frac{h(s, \tilde{v}(s)) x(s) \tilde{v}(s)\left(k_{2}-k_{3}\right)^{2}}{N k_{1} k_{2}} \frac{\rho^{2}(s)}{\rho^{\prime}(s)},
$$

and the result follows directly from the sign of the right member of the expression above.

In order to tackle the controllability of the singular arc, it can be convenient to replace $\tilde{v}(s)$ by its expression in (26). First, we have:

$$
\left\{\begin{array}{l}
-k_{2}\left(\frac{N}{\tilde{v}(s)}+s_{i n}-s\right)=\frac{k_{1}\left(s_{i n}-s\right)}{s\left(k_{1}+s\right)}-\left(s_{i n}-s\right) k_{3} \\
h(s, \tilde{v}(s))=\mu_{m} \frac{s}{k_{1}+s} e^{-k_{3} s_{i n}+k_{1} \frac{s_{i n}-s}{s\left(k_{1}+s\right)}} \\
\frac{x \tilde{v}(s)}{s_{i n}-s}=\frac{M}{s_{i n}-s}+\tilde{v}(s)=\frac{1}{s_{i n}-s}\left(M-N \frac{k_{2} s\left(k_{1}+s\right)}{\left(k_{2}-k_{3}\right) \rho(s)}\right)
\end{array}\right.
$$

therefore, the singular control becomes:

$$
\begin{aligned}
& \tilde{u}(s)=\mu_{m} \frac{s}{\left(k_{1}+s\right)\left(s_{i n}-s\right)} e^{-k_{3} s_{i n}+k_{1} \frac{s_{i n}-s}{s\left(k_{1}+s\right)}} \\
& \left(M-N \frac{k_{2} s\left(k_{1}+s\right)}{\left(k_{2}-k_{3}\right) \rho(s)}\right)\left(1+\frac{k_{2}-k_{3}}{k_{1}} \frac{s\left(k_{1}+s\right) \rho(s)}{\left(s_{i n}-s\right) \rho^{\prime}(s)}\right)
\end{aligned}
$$

In order to be admissible the singular arc must be such that $\tilde{v}(s) \geq 0$ and $\tilde{u}(s) \in[0,1]$. From $(24)$ and (26), it follows that if

$$
-N\left(k_{2}-k_{3}\right) \rho(s)>0
$$

and

$0 \leq \frac{h(s, \tilde{v}(s)) x(s) \tilde{v}(s)}{s_{i n}-s}\left(1-\tilde{v}(s) \frac{\left(k_{2}-k_{3}\right)^{2}}{N k_{1} k_{2}} \frac{\rho^{2}(s)}{\rho^{\prime}(s)}\right) \leq 1$,

then the singular control is admissible. We now make the following assumption on the system:
Hypothesis 1 The singular arc is always controllable, that is $\tilde{u}(s) \in[0,1]$.

Remark 3 Following [9],[8] and (23), it is standard to assume that

$\max _{s} \frac{x(s) \tilde{v}(s) h(s, \tilde{v}(s))}{s_{i n}-s}<1$,

where the maximum is taken for $s \in\left(0, s_{i n}\right)$ such that $\tilde{v}(s) \leq v_{m}$. This condition ensures that $\dot{s}>0$ along the singular arc in (2) whenever $u=1$. As we have $\dot{s}<0$ whenever $u=0$, one can infer that in some cases $(29)$ implies that the singular arc is controllable. However, from (26), this condition is not sufficient to define admissible singular arcs (as in the case where $N=0$ ). For instance, when $N>0$, condition (29) ensures only that $\tilde{u}(s) \leq 1$, but $\tilde{u}(s) \geq 0$ is not guaranteed.

Finally, we have the following result on the optimality of a singular arc.

Proposition 3 If a singular arc is admissible, then it satisfies Legendre-Clebsch condition (15).

Proof From (26), we obtain:

$$
\frac{\partial}{\partial u} \frac{d^{2}}{d t^{2}} H_{u}=\lambda_{s} x \mu_{m} \frac{e^{-k_{2} p-k_{3} s}}{v\left(k_{1}+s\right)} \gamma(s, v),
$$

where $\gamma(s, v):=\frac{k_{1} k_{2} N \rho^{\prime}(s)\left(s_{i n}-s\right)}{\left(k_{2}-k_{3}\right) v^{2} \rho(s)\left(k_{1}+s\right)}$. As we have $\lambda_{s}<0$ in $\mathcal{D}^{\prime}$, we obtain that $\frac{\partial}{\partial u} \frac{d^{2}}{d t^{2}} H_{u} \geq 0$ if and only if

$-N\left(k_{2}-k_{3}\right) \rho(s) \geq 0$,

which is exactly saying that $\tilde{v}(s) \geq 0$. Therefore, the singular arc satisfies Legendre-Clebsch condition.

\subsection{Optimal synthesis in the case $N \neq 0$}

Throughout this part, we assume that Hypothesis 1 is satisfied. Our aim is to perform an optimal synthesis of the problem when $N \neq 0$ and to find an optimal feeding strategy for any initial condition in $\mathcal{D}^{\prime}$

In order to determine the optimal feeding strategy, we consider the following cases:

- Case 1: $N<0$,

- Case 2: $N>0$ and $k_{2}-k_{3}>0$,

- Case 3: $N>0, k_{2}-k_{3}<0$, and $\bar{s} \geq s_{i n}$,

- Case 4: $N>0, k_{2}-k_{3}<0$, and $\bar{s}<s_{i n}$.

For each case, we can now provide the optimal synthesis:

Property 2 For Case 1, the optimal strategy is the singular arc strategy $\tilde{v}(s)$ (see Fig. 2), defined as follows: 
- if $s>\tilde{v}^{-1}(v)$ or $v=v_{m}$, then $u=0$.

- if $v=\tilde{v}(s)$ and $v<v_{m}$, then $u=\tilde{u}(s, v)$.

- if $s<\tilde{v}^{-1}(v)$ and $v<v_{m}$, then $u=1$.

Proof If $k_{2}-k_{3}>0$, then $\tilde{v}(s)$ is positive and increasing on $\left(0, s_{i n}\right)$. If $k_{2}-k_{3}<0$, we have two subcases: if $\bar{s} \geq s_{i n}$, then $\tilde{v}(s)$ is also positive and increasing on $\left(0, s_{i n}\right)$ while if $\bar{s}<s_{i n}$, then $\tilde{v}(s)$ is positive and increasing on $(0, \bar{s})$, and negative on $\left(\bar{s}, s_{i n}\right)$ (one has $\tilde{v}(s) \rightarrow+\infty$ when $s \rightarrow \bar{s})$. From Remark 2, one can check that $\psi(s, v)>0$ for $s<\tilde{v}^{-1}(v)$ and $\psi(s, v)<0$ for $s>\tilde{v}^{-1}(v)$. Consequently, Proposition 1 implies that the optimal feeding strategy is the singular arc strategy $\tilde{v}(s)$.

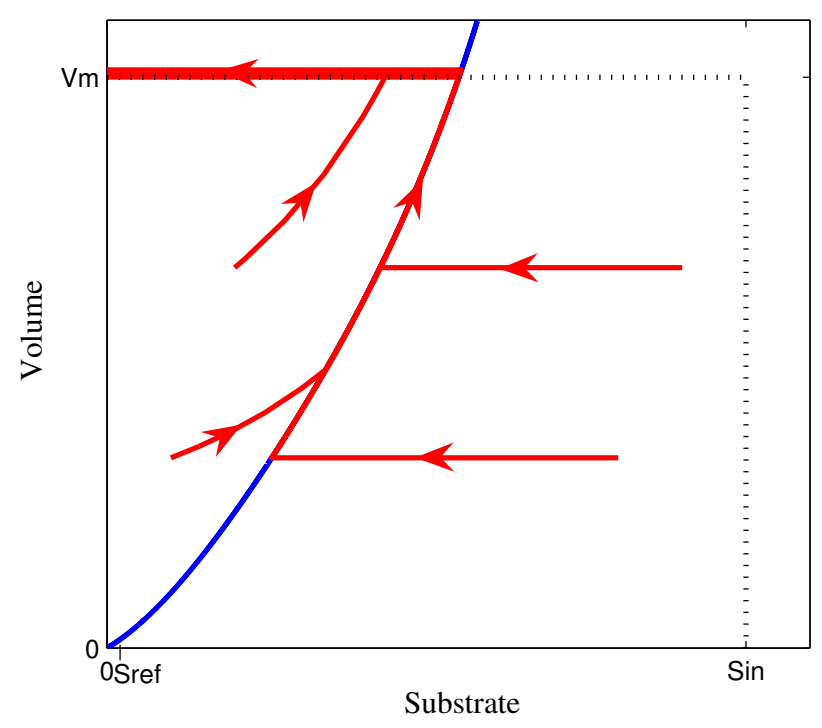

Fig. 2 Optimal trajectories (in red) for various initial conditions for the Jin growth rate, Case 1 (see Property 2). In blue, the curve $s \longmapsto \tilde{v}(s)$. Parameter values used for simulation are given in Table 1 .

Table 1 Parameter values used for simulations with the Jin growth rate (see Fig. 1, 2, and 3)

\begin{tabular}{lllllllll}
\hline & $v_{m}$ & $s_{\text {in }}$ & $s_{\text {ref }}$ & $M$ & $N$ & $k_{1}$ & $k_{2}$ & $k_{3}$ \\
\hline$N=0$ & 0.3 & 100 & 1 & 20 & 0 & 20 & 0.01 & 0.02 \\
Case 1 & 0.3 & 100 & 1 & 20 & -10 & 20 & 0.02 & 0.01 \\
Case 4 & 0.5 & 100 & 1 & 20 & 10 & 36 & 0.1 & 0.2 \\
\hline
\end{tabular}

Property 3 For Cases 2 and 3, the optimal strategy is fill and wait.
Proof We have $\tilde{v}(s)<0$ for $s \in\left(0, s_{i n}\right)$, so a singular arc is not possible and the optimal control is bang-bang. Given that $\psi(s, v)>0$ in the domain $\mathcal{D}$, we conclude the proof by using Proposition 1 .

For Case $4, \tilde{v}(s)$ is negative on the interval $(0, \bar{s})$, and positive on $\left(\bar{s}, s_{i n}\right)$ with two vertical asymptotes for $s=\bar{s}$ and $s=s_{\text {in }}$ (see Fig. 3). The next Lemma on the behavior of $\tilde{v}(s)$ is rather technical and its proof is provided in the Appendix.

Lemma 3 The function $s \longmapsto \tilde{v}(s)$ admits a unique minimum on the interval $\left(\bar{s}, s_{i n}\right)$ that we call $\left(s_{d}, v_{d}\right)$.

If $v_{d}>v_{m}$, one can easily show that the optimal strategy is fill and wait (in this case, the singular arc is not admissible), so we will only consider the case $v_{d}<v_{m}$. In order to define the optimal strategy, we divide the domain $\mathcal{D}$ in five regions A, B, C, D, E (see Fig. 3), delimited by the following curves:

- the blue curve is the mapping $s \longmapsto \tilde{v}(s)$ on $\left(\bar{s}, s_{\text {in }}\right)$,

- the solution of (2) with $u=1$ which passes through the point $\left(s_{d}, v_{d}\right)$ is denoted by $s \longmapsto \gamma_{1}(s)$ in the plane $(s, v)$. We call $L_{1}$ this curve (depicted in green on Fig. 3).

- the dot-dashed curve $L_{2}$ is the set of points $\hat{s}(v)>$ $s_{0}(v)$ for $v>v_{d}$ such that:

$$
\int_{s_{0}(v)}^{\hat{s}(v)} \frac{\psi(s, v)}{h(s, v)^{2}\left(M / v+s_{\text {in }}-s\right)} d s=0,
$$

where $s_{0}(v)$ is such that $v=\tilde{v}\left(s_{0}\right)$ and $s_{0}(v)<s_{d}$. - the solution of (2) with $u=1$ which passes through the intersection between $L_{2}$ and the line $v=v_{m}$ is denoted by $s \longmapsto \gamma_{3}(s)$ in the plane $(s, v)$. We call $L_{3}$ this curve (depicted in green on Fig. 3).

We can now define the regions as follows:

$$
\begin{aligned}
& A=\{(s, v) \in \mathcal{D} \mid v>\tilde{v}(s)\} \\
& B=\left\{(s, v) \in \mathcal{D} \backslash A \mid v>\gamma_{1}(s)\right\} \\
& D=\left\{(s, v) \in \mathcal{D} \mid v<\gamma_{3}(s)\right\} \\
& E=\left\{(s, v) \in\left[s_{d}, s_{i n}\right] \times\left[v_{d}, v_{m}\right] \mid \hat{s}^{-1}(v)<v<\tilde{v}(s)\right\} \\
& C=\{(s, v) \in \mathcal{D} \backslash(A \cup B \cup D \cup E)\}
\end{aligned}
$$

Notice that from Remark 2 we have $\psi(s, v)>0$ for $(s, v) \in A$, and $\psi(s, v)<0$ otherwise.

Remark 4 Given the definition of $\hat{s}(v)$, the curve $L_{2}$ starts at the point $\left(s_{d}, v_{d}\right)$. Nevertheless, it is not clear that $\hat{s}(v)$ will always exist for all $v \in\left[v_{d}, v_{m}\right]$. In this case, the curve $L_{2}$ will end at $s=s_{\text {in }}$ for some volume $v \in\left(v_{d}, v_{m}\right)$, and the region $\mathrm{D}$ will not exist (but the same optimal synthesis holds). 
Hypothesis 2 For any volume $v \in\left(v_{d}, v_{m}\right)$, we have $\frac{d \hat{s}(v)}{d v}>\frac{s_{i n}-\hat{s}(v)}{v}$.

If this hypothesis holds, then any trajectory starting in E can not go in $C \cup D$. According to various numerical simulations, this assumption seems to be always true. Table 2 presents a numerical verification of this hypothesis with the parameter set used for simulations (given in Table 1).

Table 2 Numerical verification of Hypothesis 2 with the parameter set used for simulations (given in Table 1).

\begin{tabular}{llllll}
\hline$v$ & $v_{d}=0.17$ & 0.2 & 0.3 & 0.40 & $v_{m}=0.5$ \\
\hline$\frac{d \hat{s}(v)}{d v}$ & $2.1 \mathrm{e} 3$ & 605 & 132 & 71 & 40 \\
$\frac{s_{i n}-\hat{s}(v)}{v}$ & 330 & 238 & 86 & 42 & 23 \\
\hline
\end{tabular}

Property 4 For Case 4 under hypothesis 2, we have: (i). If $\left(s_{0}, v_{0}\right) \in A \cup B \cup E$, the optimal strategy is the singular arc strategy $\tilde{v}(s)$, defined as follows:

- if $\left(s_{0}, v_{0}\right) \in A$, then $u=0$.

- if $v_{0}=\tilde{v}\left(s_{0}\right)$ with $s_{0} \leq s_{d}$, then $u=\tilde{u}$.

- if $\left(s_{0}, v_{0}\right) \in B$, then $u=1$.

(ii). If $\left(s_{0}, v_{0}\right) \in C$, the optimal strategy is $u=1$ until reaching $L_{2}$, and then the singular arc strategy $\tilde{v}(s)$.

(iii). If $\left(s_{0}, v_{0}\right) \in D$, the optimal strategy is fill and wait.

Proof First, let us prove (i) for $\left(s_{0}, v_{0}\right) \in A \cup B$. The region $A \cup B$ is invariant: any trajectory starting in $A \cup B$ will stay in it. Indeed, if we consider the trajectory $u=1$ starting at $(s, v)$ such that $s>s_{d}$ and $v=\tilde{v}(s)$, we have:

$$
\frac{\partial v}{\partial s}>\frac{v}{s_{i n}-s}>\frac{\partial \tilde{v}}{\partial s}
$$

which proves that $A \cup B$ is invariant. Moreover, from Lemma 2, a trajectory cannot follow the singular arc for $s>s_{d}($ as $\dot{s}<0$ for $N>0$ and $\dot{v} \geq 0)$. Given that $\psi(s, v)<0$ in $A$ and $\psi(s, v)>0$ in $B$, we can apply Proposition 1 and conclude that, if $\left(s_{0}, v_{0}\right) \in A \cup B$, then the singular arc strategy is optimal.

For proving (i), it remains to consider the case where the initial condition is in $E$. First, consider a sequence $u=0$ (at a given constant volume $v$ ) on a time interval $\left[t_{0}, t_{1}\right]$. We have for all $t \in\left[t_{0}, t_{1}\right]$ :

$\lambda_{s}(t)=\frac{-1}{h(s, v) x}, \quad \dot{\phi}(t)=\frac{-\psi(s, v)}{h(s, v)}$.
Take $v>v_{d}$, and let $s\left(t_{0}\right), s\left(t_{1}\right)$ the two substrate concentrations such that $s\left(t_{0}\right)=\hat{s}(v), v=\tilde{v}\left(s\left(t_{1}\right)\right)$, and $s\left(t_{1}\right)<s_{d}$. We obtain from $(31)$ :

$$
\int_{t_{0}}^{t_{1}} \dot{\phi}(t) d t=\int_{s\left(t_{0}\right)}^{s\left(t_{1}\right)} \frac{\psi(s, v)}{h(s, v)^{2}\left(M / v+s_{\text {in }}-s\right)} d s=0
$$

Therefore, a sequence $u=0$ that contains two switches at $t_{0}$ and $t_{1}$ is candidate for optimality.

Finally, take $\left(s_{0}, v_{0}\right) \in E$. As we have in this region $\dot{\phi}<0$, we only have two candidates $C_{1}$ and $C_{2}$ for optimality:

- $C_{1}$ : if $\phi\left(t_{0}\right)<0$, then $u=0$ until reaching $A$.

$-C_{2}$ : if $\phi\left(t_{0}\right)>0$, then the trajectory starts with $u=1$. In order to reach the target, this trajectory must switch at a time $t_{1}\left(\right.$ with $\left.\phi\left(t_{1}\right)=0\right)$. Then, it satisfies $u=0$ until reaching $A$ (as $\dot{\phi}<0$, only one switch is possible in $E$ ).

For both strategies, we must have $u=0$ until reaching the singular arc $\tilde{v}$ at a time $t_{2}$ with $\phi\left(t_{2}\right)=0$ and a substrate concentration $s\left(t_{2}\right)<s_{d}$ (see above in region $A)$. However, the second trajectory $C_{2}$ satisfies $s\left(t_{1}\right)<$ $\hat{s}\left(v\left(t_{1}\right)\right)$, hence we have

$$
\begin{aligned}
\phi\left(t_{2}\right) & =\int_{t_{1}}^{t_{2}} \dot{\phi}(t) d t=\int_{s\left(t_{1}\right)}^{s\left(t_{2}\right)} \frac{\psi(s, v)}{h(s, v)^{2}\left(M / v+s_{i n}-s\right)} d s \\
& >\int_{s\left(t_{1}\right)}^{\hat{s}(v)} \frac{\psi(s, v)}{h(s, v)^{2}\left(M / v+s_{i n}-s\right)} d s=0,
\end{aligned}
$$

where $v=v\left(t_{1}\right)=v\left(t_{2}\right)$. Thus, we get a contradiction. Therefore, the first candidate $C_{1}$ is optimal, which concludes the proof of (i).

Now consider (ii) and (iii) (i.e. let $\left(s_{0}, v_{0}\right) \in C \cup D$ a given initial condition at time $\left.t_{0}\right)$. First, if $v_{0}<v_{d}$, we have $u\left(t_{0}\right)=1$. Otherwise, we would have $\phi\left(t_{0}\right)<0$ and $u\left(t_{0}\right)=0$, but as $\dot{\phi}(t)<0$, the trajectory would not reach the target (as the control cannot switch). Secondly, assume $v_{d}<v_{0}<v_{m}$. If $u\left(t_{0}\right)=0$, then the trajectory must switch at a time $t_{1}$ (in order to reach the target). As $\dot{\phi}<0$ in $C \cup D \cup E$, the switch should be in $A$. Following the proof of (i), the trajectory will switch to the singular arc at a time $t_{1}$ such that $\tilde{v}\left(s\left(t_{1}\right)\right)=v\left(t_{0}\right)$ with $s\left(t_{1}\right)<s_{d}$. But we have:

$$
\begin{aligned}
\phi\left(t_{1}\right) & =\phi\left(t_{0}\right)+\int_{t_{0}}^{t_{1}} \dot{\phi}_{u=0}(s, v) d t \\
& <\int_{\hat{s}\left(v\left(t_{0}\right)\right)}^{s\left(t_{1}\right)} \frac{\psi(s, v)}{h(s, v)^{2}\left(M / v+s_{i n}-s\right)} d s=0
\end{aligned}
$$

as $s\left(t_{0}\right)>\hat{s}\left(v\left(t_{0}\right)\right)$, which is a contradiction. Therefore, we have $u\left(t_{0}\right)=1$ and a switch is possible only in the two following cases: 
- If $\left(s\left(t_{0}\right), v\left(t_{0}\right) \in C\right.$, then the trajectory switches for $s=\hat{s}(v)$,

- If $\left(s\left(t_{0}\right), v\left(t_{0}\right) \in D\right.$, then the trajectory switches at volume $v=v_{m}$.

This concludes the proof.

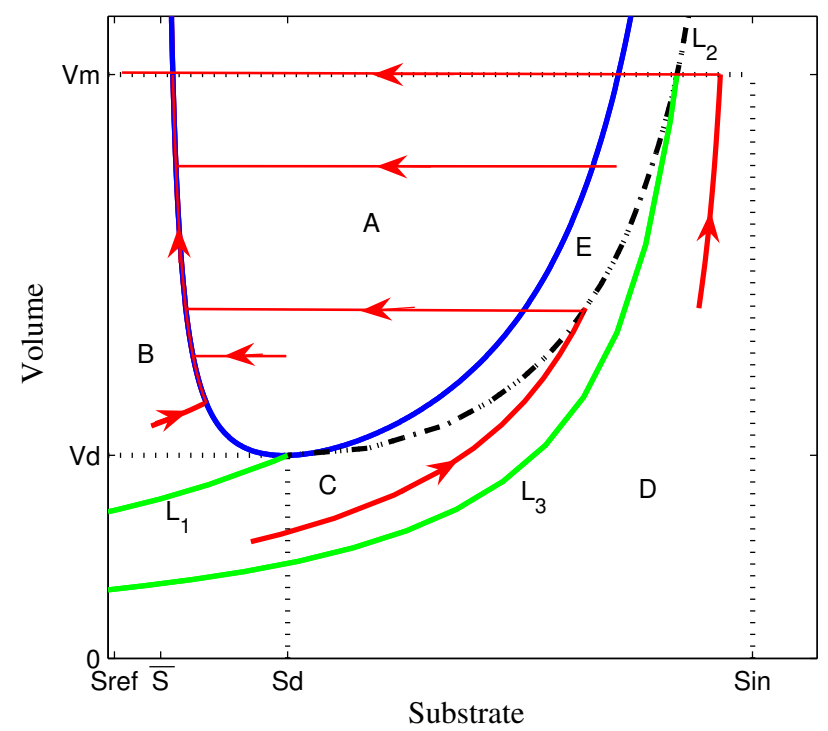

Fig. 3 Optimal trajectories (in red) for various initial conditions for the Jin growth rate, Case 4 (see Property 4 and the paragraph above this property for the definition of the curves $\left.L_{i}\right)$. In blue, the curve $\tilde{v}(s)$. Parameter values used for simulation are given in Table 1.

\section{Practical implementation}

Given model uncertainties that arise in bioprocesses and the lack of online sensors, the practical implementation of such optimal strategies is not straightforward. The first challenge is to determine which case applies since it depends on model parameters and initial conditions which are generally poorly known. Then, a robust approach should be used to implement the optimal strategy. For inhibition by product only (Section 3 ) and inhibition by product and substrate with $N=0$ (Section 4.1), the optimal strategy is either fill and wait (which implementation is straightforward), either a singular strategy which consists at regulating $s=\bar{s}$, i.e. maintaining the specific growth rate at its maximum. Implementation of the second strategy has been tackled in the case of nonmonotonic growth rate by Moreno et al. [19]. Their method - called Event-Driven Time Optimal Control (ED-TOC) - consists in the approximation of the singular arc by a sequence of bang-bang arcs (that is $u$ is equal either to 0 or 1 ) where the switching instants are determined by the variations of the specific growth rate (which is estimated via online measurement of the dissolved oxygen concentration). This strategy has been validated experimentally with the removal of the toxic organic compound 4-chlorophenol in a labscale bioreactor. Other methods have been proposed for nonmonotonic growth rate such as adaptive extremum seeking [20] but their experimental implementation has not yet been carried out (probably because of a higher complexity). Thereby, the ED-TOC strategy seems to be a good candidate for the practical implementation of the optimal strategy for $N=0$. This case is of particular interest since $N$ tends to zero when repeated fed-batch cultures are carried out (assuming that the new fed-batch culture starts, after a partial discharge of the reactor, with the substrate and product concentrations reached at the end of the previous culture).

For $N \neq 0$, the optimal trajectory (see Section 4.2) should follow the singular arc path $\tilde{v}(s)$ defined by $\psi=$ 0 . Implementation of this strategy is more problematic given the uncertain framework. Nevertheless, note that $\psi$ (see Equation (10)) is actually the directional derivative of the specific growth rate $h(s, v)$ along the vector $\left(\frac{s_{i n}-s}{v}, 1\right)$ in the $(s, v)$ plan. This vector defines the trajectory $u=1$ if we assume $\frac{u\left(s_{i n}-s\right)}{v} \gg h(s, v) x$ (as it is done in $[10,9]$ for impulsive control). Thus, along a sequence $u=1$, the observed growth rate goes through a maximum when the trajectory cross the curve $\tilde{v}(s)$. This is a first hint that an ED-TOC strategy can be adapted to this case: the optimal trajectory can be approximated by a bang-bang strategy where the variation of the growth rate determines the switching instants. Fig. 4 illustrates this approach. For each intersection between the trajectory with $u=1$ and the curve $\tilde{v}(s)$ (at times $t_{A}$ and $t_{B}$ ), the growth rate goes through a maximum. Nevertheless, a slight lag appears, probably due to the fact that the hypothesis $\frac{u\left(s_{i n}-s\right)}{v} \gg$ $h(s, v) x$ is not verified. In practice, the maximal feeding rate (taken as 1 in this work) should be tuned adequately: a high value will make the hypothesis valid, but the substrate concentration will increase rapidly during a sequence $u=1$ and the trajectory will go away from the singular arc, before the controller reacts.

In order to approximate the optimal trajectory, the following strategy can be used: 

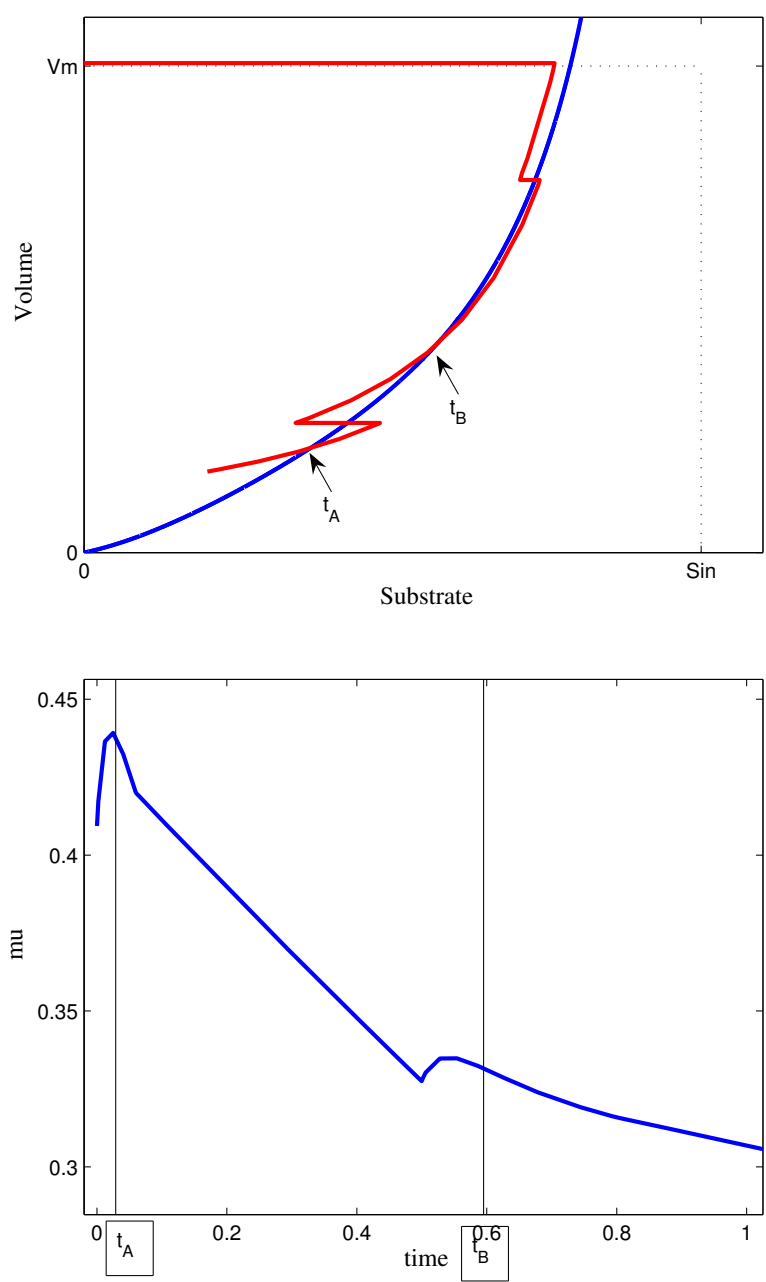

Fig. 4 Approximation of the optimal strategy for the Jin growth rate, Case 1 by a sequence of bang-bang arcs. Top: trajectory (in red) in the $(s, v)$ plan. Down: Variation of the (observed) growth rate. The intersections of the trajectory $u=1$ with the curve $\tilde{v}(s)$ (in blue) at time $t_{A}$ and $t_{B}$ can be estimated by the variation of the growth rate (see Section 5 ).

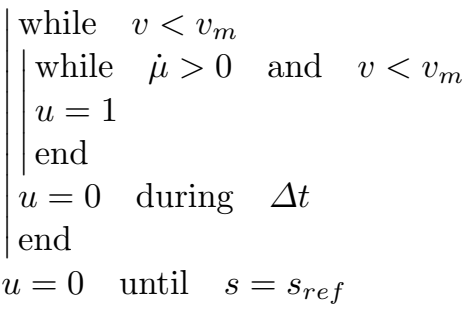

Another difficulty with this strategy is that during a sequence $u=0$, we cannot determine if the trajectory has crossed the singular arc $\tilde{v}(s)$. Nevertheless, if the control switches to $u=1$ before crossing the singular arc, then we will have $\dot{\mu}<0$, so the control will switch back to $u=0$. In practice, the time $\Delta t$ should be pre- determined via simulations and ideally adjusted by an adaptive strategy. This will deserve further investigations.

\section{Controllability assumption}

So far, we have assumed that the singular arc is always controllable. This means that the singular control always satisfies the bound constraints in the invariant domain of the system. This is a classical assumption in this kind of problem (see e.g. [8]). Nevertheless, for some initial conditions, this should not be true (as the expressions providing the singular control do not necessarily define an admissible control), and it changes the optimal synthesis. We will discuss the validity of this assumption for the different cases where a singular arc is possible (case $N=0$, case 1 and 4 of section 4.3 ) by studying if the singular control is admissible.

\subsection{Study of case $N=0$}

Let us define a volume $v^{*}$ such that:

$v^{*}=\frac{1}{h_{0}(\bar{s})}-\frac{M}{s_{i n}-\bar{s}}$.

From (22), it follows that the singular arc is controllable for $v \in\left(0, v^{*}\right]$ (indeed, if $v>v^{*}$, then $\bar{u}$ no longer belongs to $[0,1])$. Thus, the controllability condition (23) rewrites:

$$
v_{m}<v^{*}
$$

Otherwise, a trajectory cannot follow the singular arc until the maximal volume $v_{m}$, which affects the optimal synthesis. In particular, we have depicted a result which goes against intuition: it is not optimal to stay as long as possible on the singular arc (manuscript under preparation). This result applies also for the more classical problem of a growth rate $s \longmapsto \mu(s)$ with one unique maximum $\bar{s}$ (e.g. the Haldane function) and where the singular arc is precisely $s(t)=\bar{s}$.

\subsection{Study of case 1}

We now consider the first case of Section 4.3 when $N<0$, and we study in particular if (26) defines an admissible control. The values of the parameter are taken from Table 1 . In particular we have $k_{2}-k_{3}>0$, thus we get from $(24)$ that $s \longmapsto \tilde{v}(s)$ is well defined and positive on $\left[0, s_{i n}\right)$. Moreover, we have that $\tilde{v}(s)$ goes to infinity when $s$ goes to $s_{i n}$. From (26), we have that $\tilde{u}(s) \geq 0$ (as $N<0)$. But the inequality $\tilde{u}(s) \leq 1$ may not be valid 
for all $s \in \mathcal{D}^{\prime}$. In view of the expressions of the singular control $\tilde{u}(s)$ of Section 4.2, finding the set of points where this inequality is satisfied is more difficult than for the case $N=0$. From a numerical point of view, the plot of $s \longmapsto \tilde{u}(s)$ (see Fig. 5) shows that the singular arc is controllable only if $s$ is in some interval $\left[s_{1}, s_{2}\right]$, where $s_{1}$ and $s_{2}$ are such that $\tilde{u}\left(s_{1}\right)=\tilde{u}\left(s_{2}\right)=1$, see Fig. 5.
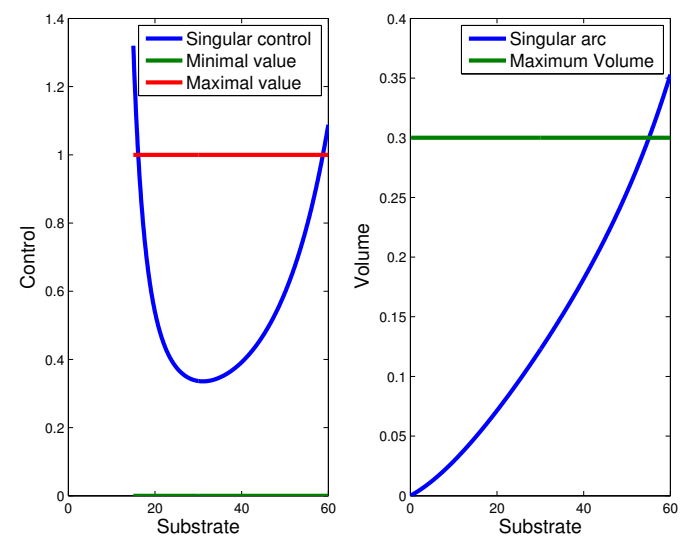

Fig. 5 Figure left : plot of the singular control $s \longmapsto \tilde{u}(s)$ defined by (26) when $N<0$. Figure right: plot of the singular arc $s \longmapsto \tilde{v}(s)$ defined by $(24)$ when $N<0$.

Therefore, in this case, our optimal synthesis for the trajectories bang-singular-bang remains valid under two conditions:

- the trajectory should reach the singular arc $\tilde{v}(s)$ with $s>s_{1}$,

- the trajectory can follow the singular arc until the maximal volume, that is $\tilde{v}\left(s_{2}\right)>v_{m}$.

\subsection{Study of case 4}

Recall that in case 4 of Section 4.3 , we have $N>0$, $k_{2}-k_{3}<0$ and $\bar{s}<s_{i n}$. Contrary to the case 1 , we have the following result.

Proposition 4 Assume that (29) holds. Then for all $s \in\left(\bar{s}, s_{d}\right]$, the singular control $\tilde{u}(s)$ given by $(26)$ satisfies $\tilde{u}(s) \in[0,1]$.

The proof is technical and is given in the Appendix.

\section{Conclusion}

This paper has tackled the minimal time control problem for fed-batch bioreactors, in presence of an inhibitory product, which is released by the biomass proportionally to its growth. Thanks to Pontryagin maximum principle, Green's theorem, and properties of the switching function, we have provided the optimal strategy, which is of type bang-bang or bang-singular-bang depending on the parameter set. Finally, we have provided a methodology in order to implement these strategies in a real process.

\section{Appendix}

We first prove Lemma 3. Let us define $\beta:=\frac{k_{1}}{k_{2}-k_{3}}<0$ and write $\tilde{v}(s)$ as follows:

$$
\tilde{v}(s)=-\frac{N k_{2}}{k_{2}-k_{3}} \frac{\rho(s)-\beta}{\rho(s)\left(s_{i n}-s\right)} .
$$

Let us now show that the derivative of the mapping $s \longmapsto \alpha(s):=\frac{\rho(s)-\beta}{\rho(s)\left(s_{i n}-s\right)}$ has exactly one zero on the interval $\left(\bar{s}, s_{i n}\right)$. By derivating, one has:

$$
\alpha^{\prime}(s)=\frac{\gamma(s)}{\rho^{2}(s)\left(s_{i n}-s\right)^{2}},
$$

where

$$
\gamma(s)=\rho^{2}(s)-\beta \rho(s)+\beta \rho^{\prime}(s)\left(s_{\text {in }}-s\right) .
$$

Now, $s \longmapsto \gamma(s)$ is convex on $\left[\bar{s}, s_{i n}\right]$ as we have

$$
\gamma^{\prime \prime}(s)=2 \rho^{\prime}(s)^{2}+4 \rho(s)-6 \beta>0 .
$$

Finally, one has $\gamma(\bar{s})<0$ and $\gamma\left(s_{i n}\right)>0$, therefore $\gamma$ admits exactly one zero on $\left(\bar{s}, s_{i n}\right)$. It follows that $\alpha$ has also exactly one zero on $\left(\bar{s}, s_{i n}\right)$ which concludes the proof as $\lim \tilde{v}(s)=+\infty$ when $s$ goes to $\bar{s}$ or to $s_{i n}$.

We now prove that for case 4 (that is when $N>0$ and $\left.k_{2}-k_{3}<0\right)$, the singular control $\tilde{u}$ satisfies $\tilde{u}(s) \in$ $[0,1]$ for $s \in\left(\bar{s}, s_{d}\right]$. Using $(29)$ and $N>0$, we get easily that $\tilde{u}(s) \leq 1$. Let us now show that $\tilde{u}(s) \geq 0$ for $s \in\left(\bar{s}, s_{d}\right]$. By $(26)$, it is enough to prove that

$$
\varphi(s):=\tilde{v}(s) \frac{\left(k_{2}-k_{3}\right)^{2} \rho^{2}(s)}{N k_{1} k_{2} \rho^{\prime}(s)} \leq 1 .
$$

By (25), the derivative of $\varphi$ can be written:

$\varphi^{\prime}(s)=\frac{\varphi(s)-1}{s_{i n}-s}+\lambda(s) \varphi(s)$,

where

$$
\lambda(s):=\frac{2\left[\rho^{\prime}(s)^{2}-\rho(s)\right]}{\rho(s) \rho^{\prime}(s)} .
$$

As $\beta<0$, it is straightforward to check that $\lambda(s)>0$ for $s \in\left(\bar{s}, s_{d}\right]$. Now, as $s_{d}$ is the unique minimum of $\tilde{v}$ on $\left(\bar{s}, s_{i n}\right)$, one has $\frac{\partial \tilde{v}}{\partial s}\left(s_{d}\right)=0$, and by $(25)$, this gives 
$\varphi\left(s_{d}\right)=1$. It follows from $(34)$ that $\varphi^{\prime}\left(s_{d}\right)>0$, thus $\varphi$ is increasing in a neighborhood of $s_{d}$. As a consequence, we have $\varphi \leq 1$ in a left neighborhood of $s_{d}$.

Assume now by contradiction that $\varphi-1$ is vanishing on the interval $\left(\bar{s}, s_{d}\right)$, and consider the greatest $s_{0}<$ $s_{d}$ such that $\varphi\left(s_{0}\right)=1$. By definition of $s_{0}$, we have $\varphi<1$ on the interval $\left(s_{0}, s_{d}\right)$, consequently we get that $\varphi^{\prime}\left(s_{0}\right) \leq 0$. On the other hand, (34) gives $\varphi^{\prime}\left(s_{0}\right)=$ $\lambda\left(s_{0}\right) \varphi\left(s_{0}\right)>0$ (recall that both $\lambda$ and $\varphi$ are positive on $\left.\left(\bar{s}, s_{d}\right]\right)$. Therefore, we have a contradiction which proves that we have $\varphi<1$ on the interval $\left(\bar{s}, s_{d}\right)$.

Acknowledgements This work was supported by Programa de Financiamiento Basal from the Center of Mathematical Modeling, Universidad de Chile and was developed in the context of DYMECOS INRIA associated team. F. Mairet was partially supported by FONDECYT-Chile program (N 3120117), and by INRIA Chile - CIRIC. The first author thanks the team DYMECOS for partial financial support and INRIA Sophia-Antipolis Méditerranée for providing him a research opportunity of one year at INRA-INRIA 'MODEMIC' team.

\section{References}

1. J. Lee, S.Y. Lee, S. Park, A.P.J. Middelberg, Control of fed-batch fermentations, Biotechnology advances, Vol. 17, 1, pp. 29-48, 1999.

2. C. Liu, Optimal control for nonlinear dynamical system of microbial fed-batch culture, Journal of computational and applied mathematics, 232, pp. 252-261, 2009.

3. J. A. Moreno, Optimal time control of bioreactors for the wastewater treatment, Optim. Control Appl. Meth., 20, pp. 145-164, 1999.

4. J. Monod, Recherches sur la Croissance des Cultures Bactériennes, Hermann, Paris 1942.

5. H. L. Smith, P. Waltman, The theory of the chemostat, Dynamics of microbial competition, cambridge University Press, 1995.

6. J. F. AndREws, A mathematical model for the continuous culture of microorganisms utilizing inhibitory substrates, Biotechnology Bioengineering, vol. 10, pp. 707$723,1968$.

7. A. Miele, Application of Green's Theorem to the extremization of linear integrals, Symp. on Vehicle Systems Optimization, Garden City, L. I. New York, pp. 26-35, 1961.

8. A. RApaport, D. Dochain, , Minimal time control of fed-batch processes for growth functions with several maxima, IEEE Transactions on Automatic Control, vol. 56, 11, pp. 2671-2676, 2011.

9. T. Bayen, P. Gajardo, F. Mairet, Optimal synthesis for the minimum time control problems of fed-batch bioprocesses for growth functions with two maxima, http://hal.archives-ouvertes.fr/hal-00655534/fr/, submitted to J. of Optim. Theory and Appl, 2011.

10. P. Gajardo, H. Ramirez, A. Rapaport, Minimal time sequential batch reactors with bounded and impulse controls for one or more species, SIAM J. Control Optim., Vol. 47, 6, pp. 2827-2856, 2008.

11. J. Hong, Optimal substrate feeding policy for a fed batch fermentation with substrate and product inhibition kinetics, Biotechnology and bioengineering, 28, pp. 1421-1431, 1986.
12. H.J. KelLey, A transformation approach to singular subarcs in optimal trajectory and control problems, SIAM J. on Control and Optimization, vol. 2, pp. 234-240, 1964.

13. P.M. DoRAn, Bioprocess engineering principles, Academic Press, 1995.

14. C.K. Jin, H.L. ChiAng, S.S. WANG, Steady state analysis of the enhancement in ethanol productivity of a continuous fermentation process employing a proteinphosphotifind complex as a protecting agent, Enzyme Microbiol. Technol., vol. 3, pp. 249-257, 1981.

15. L. Cesari, Optimization - Theory and Applications, Problems with Ordinary Differential Equations, Springer-Verlag, New-York, 1983.

16. B. Bonnard, J.-B. Caillau, E. Trélat, Second order optimality conditions in the smooth case and applications in optimal control, ESAIM Control Optim. Calc. Var. 13, 2, pp. 207-236, 2007.

17. H. M. Robbins, A Generalized Legendre-Clebsch Condition for the Singular Cases of Optimal Control, IBM J. of Research and Development 11, pp. 361-372, 1967.

18. D.E. Contols, Kinetics of bacterial growth: Relationship between population density and specific growth rate of continuous cultures. Journal of General Microbiology, 21, pp. 40-50, 1959.

19. J.A. Moreno, M.J. Betancur, G. Buitrón, I. MORENO-ANDRADE, Event-driven time-optimal control for a class of discontinuous bioreactors, Biotechnology and bioengineering, 94, pp. 803-814, 2006.

20. P. Cougnon, D. Dochain, M. Guay, M. Perrier, Online optimization of fedbatch bioreactors by adaptive extremum seeking control, Journal of Process Control, vol. 21, 10, pp. 1526-1532, 2011. 